\title{
Continued depression of maximal oxygen consumption and mitochondrial proteomic expression despite successful coronary artery bypass grafting in a swine model of hibernation
}

\author{
Rosemary F. Kelly, MD, ${ }^{\text {add }}$ Jesús A. Cabrera, MD, PhD, ${ }^{\text {a,d }}$ Elizabeth A. Ziemba, MD, ${ }^{\text {a }}$ Melanie Crampton, ${ }^{\mathrm{b}}$ \\ Lorraine B. Anderson, $\mathrm{PhD},{ }^{\mathrm{c}}$ Edward O. McFalls, MD, ${ }^{\mathrm{b}, \mathrm{d}}$ and Herbert B. Ward, MD, $\mathrm{PhD}^{\mathrm{a}, \mathrm{d}}$
}

\begin{abstract}
Objective: Clinical studies indicate incomplete functional recovery of hibernating myocardium after coronary artery bypass grafting. We hypothesized that persistent contractile abnormalities after coronary artery bypass grafting are associated with decreased mitochondrial proteins involving electron transport chain that might limit maximal oxygen consumption.
\end{abstract}

\begin{abstract}
Methods: Seven pigs with hibernating myocardium underwent off-pump revascularization with left internal thoracic artery to mid left anterior descending artery. At 4 weeks, left internal thoracic artery anastomosis was patent by multidetector computed tomography. Regional function (transthoracic echocardiography) and blood flow (microspheres) were assessed at rest and during high-dose dobutamine $(40 \mu \mathrm{g} /[\mathrm{kg} \cdot \min ])$. Expression of electron transport chain proteins was analyzed with isobaric tags for relative and absolute quantification.

Results: After revascularization, multidetector computed tomography confirmed severe left anterior descending stenosis and patent left internal thoracic artery graft. Regional function and blood flow normalized at rest; however, function in left anterior descending distribution remained depressed relative to remote regions, and myocardial blood flow in that region did not increase normally when challenged with high-work state. Concomitant with reduced maximal blood flow response in left anterior descending region was more than $40 \%$ reduction in electron transport chain proteins essential to adenosine triphosphate production.
\end{abstract}

Conclusions: Despite successful revascularization of hibernating myocardium, regional function and blood flow remained depressed during catecholamine stress. Electron transport chain proteins known to be downregulated during adaptive process within hibernating myocardium did not normalize after revascularization. These data demonstrate a potential bioenergetic cause of persistent dysfunction and heart failure within successfully revascularized hibernating myocardium. (J Thorac Cardiovasc Surg 2011;141:261-8)

Hibernating myocardium is a clinical entity that is manifested as viable but persistently dysfunctional myocardium in response to repetitive myocardial ischemia and is characterized by reduced regional blood flow without evidence of necrosis. ${ }^{1,2}$ Experimental studies indicate that several adaptations occur in response to chronic hypoperfusion, including remodeling of resistance vessels, ${ }^{3}$ increased glucose uptake, ${ }^{4,5}$ altered expression of the calcium-regulatory

\footnotetext{
From the Departments of Surgery, ${ }^{\mathrm{a}}$ Medicine, ${ }^{\mathrm{b}}$ and Biochemistry, Molecular Biology \& Biophysics, ${ }^{\mathrm{c}}$ University of Minnesota, and the Veterans Affairs Medical Center, ${ }^{\mathrm{d}}$ Minneapolis, Minn

Supported by Thoracic Surgery Foundation for Research and Education Nina Starr Braunwald Award and Veterans Affairs Merit Review Award (to R.F.K.) and National Institutes of Health grant RO1 HL089307-01A2 (to E.O.M.).

Disclosures: Authors have nothing to disclose with regard to commercial support.

Read at the 90th Annual Meeting of The American Association for Thoracic Surgery, Toronto, Ontario, Canada, May 1-5, 2010.

Received for publication May 1, 2010; revisions received Aug 20, 2010; accepted for publication Aug 27, 2010.

Address for reprints: Rosemary F. Kelly, MD, Associate Professor of Surgery, Division of Cardiothoracic Surgery, Department of Surgery, University of Minnesota, VA Medical Center, One Veterans Dr, Minneapolis MN 55417 (E-mail: kelly071@umn.edu).

$0022-5223 / \$ 36.00$

Copyright (C) 2011 by The American Association for Thoracic Surgery

doi:10.1016/j.jtcvs.2010.08.061
}

proteins, ${ }^{6,7}$ and apoptosis. ${ }^{8}$ These responses to chronic ischemia may be a coordinated response to downregulate myocardial oxygen expenditure with the reduced oxygen availability and thus ensure myocyte survival. Clinically, revascularization of hibernating myocardium results in variable degrees of contractile recovery, and this heterogeneous response may reflect the inability of the myocardium to reverse these pleomorphic adaptations in full.

Since the seminal observation by Murray and colleagues ${ }^{9}$ of ischemic myocardial preconditioning in the anesthetized canine model, mitochondria have been identified as being central to the understanding of myocardial protection. ${ }^{10}$ The chronically ischemic mitochondria within the myocyte must adapt to hypoxia resulting from decreased blood flow, with a balanced reduction in energy and reactive oxygen species production to preserve myocardium. ${ }^{11}$ As we have shown, adaptations to hibernation are multifactorial and involve alterations in the bioenergetics and proteomics of the mitochondria that allow the tissue to maintain viability but at reduced rate of energy production. ${ }^{12}$ An inability to support electron flow through the electron transport chain (ETC) may lead to inefficient oxygen consumption during high work states, and a downregulation of ETC proton 


$$
\begin{aligned}
& \text { Abbreviations and Acronyms } \\
& \text { ETC = electron transport chain } \\
& \mathrm{iTRAQ}=\text { isobaric tags for relative and absolute } \\
& \text { quantification } \\
& \text { LAD = left anterior descending coronary artery } \\
& \text { LITA }=\text { left internal thoracic artery } \\
& \text { MDCT }=\text { multidetector computed tomography } \\
& \mathrm{MS}=\text { mass spectrometry } \\
& \text { RCI = respiratory control index } \\
& \text { TTE }=\text { transthoracic echocardiography }
\end{aligned}
$$

gradient could therefore result in a negative effect on maximal oxygen expenditure and subsequently on myocardial contraction. We believe that hibernating myocardium is a coordinated proteomic response to persistent ischemic stresses that result in preserved viability at the expense of maximal energy production. ${ }^{11}$ The reversibility of these adaptations after revascularization has never been tested in suitable animal models. On the basis of the variable recoveries of hibernating myocardium in clinical studies, we postulated that mitochondrial proteomic adaptations, which limit oxidative injury and apoptosis at the expense of maximal oxygen consumption and functional recovery, may persist despite revascularization.

In a swine model of hibernating myocardium, we hypothesized that persistent myocardial wall motion abnormality in revascularized myocardium by off-pump coronary artery bypass graft $(\mathrm{CABG})$ is associated with persistently depressed expression of key mitochondrial proteins involved with the ETC.

\section{MATERIALS AND METHODS Animal Model}

All animal studies were approved by the institutional animal care and use committee of the Minneapolis VA Medical Center and conform to current National Institutes of Health (Guide for the Care and Use of Laboratory Animals, www.nap.edu/catalog/5140.html) and American Physiological Society guidelines for the use and care of laboratory animals.

\section{Operative Technique}

To create a model that reflects the clinical situation, we used a wellestablished swine model of chronic myocardial ischemia. ${ }^{4,12}$ Two survival operations and a terminal operation were performed in 7 animals during a 16-week time course. The first operation created hibernating myocardium with the placement of a left anterior descending artery (LAD) occluder at time 0 , the second operation surgically revascularized the myocardium with left internal thoracic artery (LITA) to LAD at 12 weeks, and finally the terminal procedure occurred 4 weeks after the bypass operation at 16 weeks.

Chronic hibernation model (8- to 10-kg pigs). Animals were sedated with tiletamine hydrochloride and zolazepam (Telazol, $4 \mathrm{mg} /$ $\mathrm{kg}$ intramuscularly) and xylazine ( $2 \mathrm{mg} / \mathrm{kg}$ intramuscularly), intubated, and anesthetized with isoflurane $(2 \%)$. Through a left thoracotomy approach, the chest and pericardium were opened in a sterile fashion. The
LAD was dissected free, and a plastic c-shaped ring with an internal diameter of $2 \mathrm{~mm}$ encircled the LAD proximal to the first diagonal without occluding the vessel and was secured with sutures. The thoracotomy incision was closed in layers. The animal recovered for 12 weeks. We have previously shown that this vascular constrictor does create hibernating myocardium with a reduction in regional blood flow in the LAD region with time and loss of wall thickening in the anterior wall function 12 weeks after instrumentation. $^{4,12}$ To confirm hibernating myocardium, we documented proximal LAD stenosis by multidetector computed tomography (MDCT) and wall motion abnormality by transthoracic echocardiography (TTE) before the second operation. At 12 weeks, animals underwent the second survival operation, which was revascularization of the hibernating myocardium.

Revascularization model (50- to 60-kg pigs). Animals were again sedated, intubated, and anesthetized as noted previously. Coronary artery revascularization was performed through a midline sternotomy with the LITA dissected free from the chest wall. Lidocaine $(1 \mathrm{mg} / \mathrm{kg})$ was administered before opening the pericardium. The LAD distal to the site of c-ring occlusion was then exposed. The animal was heparinized (100 unit $/ \mathrm{kg}$ ). With an off-pump technique, the LAD was stabilized with a Titan Stabilizer (Estech, San Ramon, Calif). LAD perfusion was maintained with a Flo-thru intraluminal shunt (Synovis Surgical Innovations, St Paul, Minn). A LITA anastomosis to the LAD was performed just beyond the occluder with 8-0 Prolene suture (Ethicon, Inc, Somerville, NJ). On completion of the anastomosis, the shunt and LITA occluder were removed. Protamine was given to reverse the heparin, and the chest was closed in layers. A second MDCT and TTE were done before the terminal study at 16 weeks.

Terminal procedure (70- to 80-kg pigs). Four weeks after revascularization, a terminal study was performed. Animals were sedated, intubated, anesthetized, and monitored. Femoral and carotid arterial monitoring lines were placed to monitor blood pressure, obtain blood samples, and infuse intraventricular colored microspheres. Fluorescently labeled colored microspheres were injected into the left ventricle before and after a 5-minute infusion of dobutamine $(40 \mu \mathrm{g} /[\mathrm{kg} \cdot \mathrm{min}])$ to determine regional blood flows. A resternotomy was performed to excise the heart, and tissue samples were obtained for blood flow, proteomic, and histologic analyses.

\section{Echocardiographic Analysis}

Two-dimensional TTE measured regional myocardial function in the LAD and circumflex regions of the swine heart. The circumflex region (remote) was used as the control, with measurements made at standardized locations to allow comparisons between animals. TTE with and without dobutamine infusion was performed with a Vivid 7 Ultrasound machine (GE Healthcare, New York, NY) during deep sedation without intubation. Heart rate, heart rhythm, and oxygenation were monitored during the procedure. Wall thickening was measured at the right parasternal short-axis view in the posterior (circumflex) and anterior (LAD) walls. Wall thickening was computed as the difference between end-systolic and end-diastolic wall thicknesses and expressed as a percentage of end-diastolic thickness.

\section{MDCT Analysis}

MDCT was used to document LAD stenosis and confirm LITA graft patency. MDCT scanning of the hibernating and revascularized swine models was performed with the Brilliance 40 MDCT scanner (Phillips Medical Systems, Cleveland, Ohio) during deep sedation with Telazol without designated breath hold or $\beta$-blockade. Electrocardiographically gated image acquisition was used to reconstruct images retrospectively. A volume of $150 \mathrm{~mL}$ of contrast media was injected intravenously at a rate of $4 \mathrm{~mL} / \mathrm{s}$. Scanning was triggered automatically when contrast enhancement within the descending aorta reached a threshold level of 150 Hounsfield units. At least 10 phases were reconstructed for each study, and coronary analysis was done at either $75 \%$ phase or end-systolic phase. Image analysis was done with a dedicated workstation (Philips Extended Brilliance Workspace; Phillips Medical Systems). 


\section{Blood Flow Analysis}

Myocardial blood flow was quantified with fluorescently labeled $15-\mu \mathrm{m}$ diameter microspheres injected into the left ventricle at rest and after a 5minute dobutamine $(40 \mu \mathrm{g} /[\mathrm{kg} \cdot \mathrm{min}])$ infusion at the terminal study. Forty thousand fluorescently labeled microspheres (Triton Technology Inc, San Diego, Calif) per kilogram were injected for each analysis. At the terminal study, the heart was serially sectioned, and samples of fresh tissue were rapidly excised from subendocardial and subepicardial LAD and remote territories for regional perfusion analysis. Approximately $1 \mathrm{~g}$ of tissue was digested with $4-\mathrm{mol} / \mathrm{L}$ potassium hydroxide with $1 \%$ polysorbate 80 for 72 hours. The digested material was filtered through $10-\mu \mathrm{m}$ Isopore membrane filter (Millipore Corporation, Billerica, Mass) to retain the microsphere sediments on the filter. The filters were washed in $3 \mathrm{~mL}$ diethylene glycol monoethyl ether acetate, which dissolves the microspheres and releases the fluorochrome. The fluorescent signal was then measured in a PerkinElmer LS-55 Luminescence Spectrometer (PerkinElmer Inc, Waltham, Mass). The fluorescent signal was normalized to the tissue weight, and differences between samples were analyzed.

\section{Histologic Analysis}

Tissue samples were fixed with $4 \%$ paraformaldehyde and processed as paraffin-embedded sections. For histochemical examination, sections were stained with hematoxylin-eosin and Masson trichrome. Triphenyl tetrazolium chloride stain was used to confirm viability of the myocardium.

\section{Mitochondrial Isolation}

Fresh tissue was rapidly excised from subendocardial regions of the LAD and remote territories. As previously described, myocardial tissue was placed in ice cold mitochondrial isolation buffer $(\mathrm{pH} 7.15)$ containing 50-mmol/L sucrose, 200-mmol/L mannitol, 1-mmol/L ethylene glycol tetraacetic acid. 5-mmol/L potassium hypophosphite, 5-mmol/L 3-(Nmorpholino) propanesulfonic acid, nagarse $(2 \mathrm{mg} / \mathrm{mL})$ and $0.1 \%$ fatty acid-free bovine serum albumin. Myocardium was minced, and a $5 \%$ homogenate was made. ${ }^{13}$ The supernatant was centrifuged twice, each at $8000 \mathrm{~g}$ for 10 minutes. The mitochondria were collected, and the protein concentration was determined. In general, the mitochondrial preparations contained approximately $5 \mathrm{mg}$ protein/mL, and the citrate synthase activity amounted to $2.4 \mathrm{U} / \mathrm{mg}$.

\section{Mitochondrial Respiration}

Mitochondria were suspended in mitochondrial respiration buffer composed of $110-\mathrm{mmol} / \mathrm{L}$ sucrose, $0.5-\mathrm{mmol} / \mathrm{L}$ ethylene glycol tetraacetic acid, 3-mmol/L magnesium chloride, $70-\mathrm{mmol} / \mathrm{L}$ potassium chloride, $10-\mathrm{mmol} / \mathrm{L}$ potassium dihydrogen phosphate, 20-mmol/L taurine, 20-mmol/L 4-(2-hydroxyethyl)-1-piperazineethanesulfonic acid and $0.1 \%$ fatty acid-free bovine serum albumin. They were placed into the respiration chamber equipped with a Clark electrode to measure oxygen concentrations at $25^{\circ} \mathrm{C}$. Once a steady state had been achieved, state 2 respiration was assessed by determining the rate constant for oxygen consumption in the presence of complex I (10-mmol/L glutamate and 5-mmol/ $\mathrm{L}$ malate) and complex II (10-mmol/L succinate) substrates. Once the oxygen curve was stable, $5-\mathrm{mmol} / \mathrm{L}$ adenosine diphosphate was given, and the rate constant for oxygen consumption was determined for calculation of state 3 conditions. The respiratory control index (RCI) was defined by the ratio of state 3 to state 2 .

\section{Isobaric Tags for Relative and Absolute Quantification of Mitochondrial Proteins}

Identification and relative quantification of inner matrix proteins from mitochondria that have been isolated from the swine was performed with isobaric tags for relative and absolute quantification (iTRAQ) reagents (Applied Biosystems Inc, Foster City, Calif) in conjunction with liquid chromatography and tandem mass spectrometry (MS). The iTRAQ isobaric reagents labeled all primary amines to yield labeled peptides that were identical in mass and were also identical in single MS mode. In tandem MS mode, however, they produced strong, diagnostic, low-mass signature ions in each of the 8 different samples, which allowed quantification from all 8 samples. We compared the protein concentrations from mitochondria of the LAD region for each of the revascularized animals with those from a size- and sex-matched control animal that had not undergone any surgical procedures. To extract mitochondrial proteins from individual samples, $80 \mu \mathrm{g}$ of the mitochondrial protein isolates were centrifuged and the pellet was rehydrated in $0.5-\mathrm{mol} / \mathrm{L}$ triethylammonium bicarbonate buffer $(\mathrm{pH}$ $8.5)$, reduced, alkylated, trypsin digested independently in parallel, and labeled with iTRAQ reagents to each of the samples. After labeling of the peptides, all samples were pooled and dried in vacuum before liquid chromatography and tandem MS. To reduce the complexity of the tryptic peptides, peptides were separated by a strong cation exchange into 16 fractions. The peptides from each fraction were separated by reversephase high-performance liquid chromatography and then introduced online into a mass spectrometer. ${ }^{14}$ The capillary high-performance liquid chromatography system was interfaced with QSTAR Pulsar $i$ quadrupole mass spectrometer (Applied Biosystems) with a nanoelectrospray ionization source. ${ }^{15}$ Protein identification and relative quantification were carried out with ProteinPilot version 2 software (Applied Biosystems). For relative quantification, ProteinPilot calculated the iTRAQ ratio for each reagent pair and determined an average iTRAQ ratio for each protein. ${ }^{14}$ This relative quantification was based on the ratio of the mitochondrial protein expression from the LAD region in each revascularized animal versus the mitochondrial protein expression from the LAD region of a control animal This ratio indicated whether a protein was upregulated or downregulated.

\section{Statistical Analysis}

Blood flow data were analyzed by assessing the quantity of fluorescence trapped in the $\mathrm{LAD}$ tissue at the time of injection of the microspheres relative to that in remote tissue. Means and SEs were calculated for each group. TTE and RCI data were expressed as mean \pm SE. Differences between LAD and remote regions in blood flow, TTE, and RCI analyses were tested with the Student paired $t$ test. The iTRAQ analysis used a standard protocol to compare the ratio of protein expression of isolated mitochondria between normal and revascularized myocardium. The expression of proteins was denoted as mean \pm SE. A 1-sample $t$ test analysis with a hypothesized mean of 1 was performed with the statistical software package StatView version 5.0.1 (SAS Institute Inc, Cary, NC).

\section{RESULTS}

We performed 7 hibernation surgical procedures followed by revascularization surgery in a swine model. All animals that underwent the revascularization operation survived to the terminal procedure performed 4 weeks after revascularization. There were no infections of any surgical site, and there were no animal losses, sternal complication, or myocardial infarctions during or after the revascularization surgery. Each animal served as its own control, with the LAD region compared with the circumflex artery (remote) region.

At the time of the terminal study, all animals had a patent LITA to LAD anastomosis. The anastomosis was confirmed to be patent with a $1.5-\mathrm{mm}$ probe. The occluder site was less than $1.0 \mathrm{~mm}$ probe diameter, and the LAD just distal to the occluder measured at least $2.5 \mathrm{~mm}$ in diameter in all animals. Triphenyl tetrazolium chloride staining of the myocardium beyond the LITA to LAD anastomosis confirmed viability in all 7 animals. 
TTE Analysis of Left Ventricular Function Pre-Revascularization

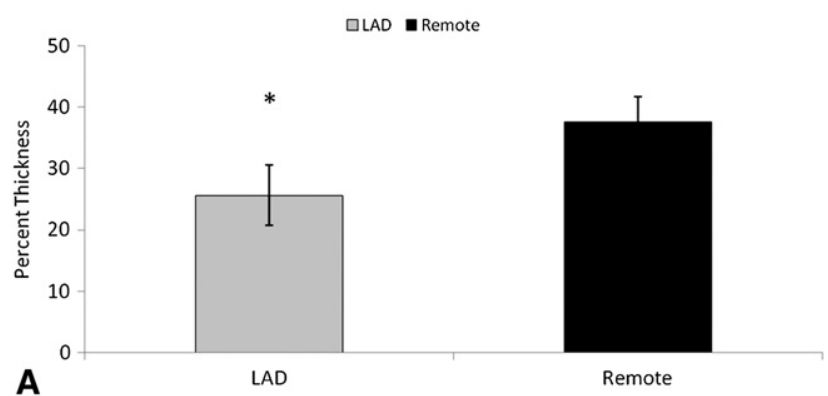

TTE Analysis of Left Ventricular Function Post-Revascularization

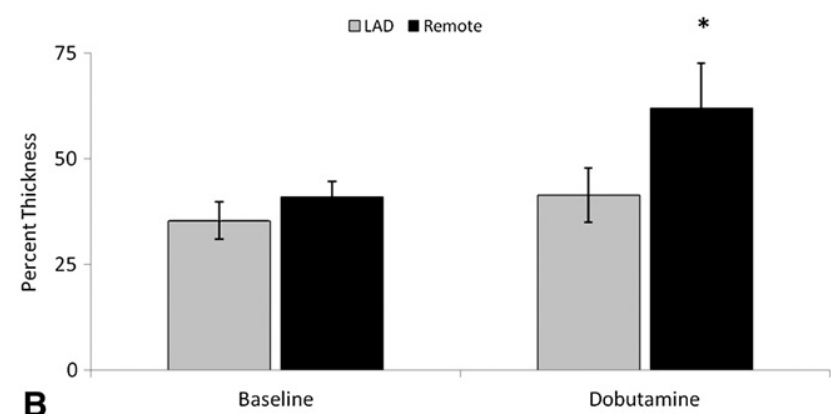

FIGURE 1. A, Transthoracic echocardiography performed at 12 weeks confirmed hibernation with reduced function in left anterior descending coronary artery $(L A D)$ region relative to remote region at baseline $(\mathrm{n}=6)$. Asterisk indicates $P<.05 . \mathrm{B}$, Transthoracic echocardiography (TTE) performed 4 weeks after revascularization of hibernating myocardium compared percentage wall thickness change of left anterior descending coronary artery $(L A D)$ territory (hibernation) with remote (control) territory. Measurements were taken from images obtained at baseline and with dobutamine infusion of $40 \mu \mathrm{g} /(\mathrm{kg} \cdot \min )$ for 5 minutes $(\mathrm{n}=7)$. Asterisk indicates $P<.05$

Myocardial function was assessed by 2-dimensional TTE done under sedation to assess regional wall abnormalities compared to remote regions. We used a method similar to our previous experience with hibernating myocardium studies. ${ }^{4}$ Twelve weeks after LAD occluder placement, animals showed evidence of hibernating myocardium, with reduced wall thickening at rest in the LAD territory (Figure 1, $A$ ). At 4 weeks after revascularization, a repeat TTE was performed. This TTE was done at rest and with a dobutamine infusion $(40 \mu \mathrm{g} /[\mathrm{kg} \cdot \mathrm{min}])$. We found that after revascularization, the baseline function of the LAD territory returned to normal function. During dobutamine infusion, however, the revascularized myocardium showed incomplete recovery of myocardial function relative to remote myocardium (Figure 1, B).

After TTE studies, cardiac-gated MDCT studies were performed at 12 weeks to assess coronary anatomy and occluder stenosis and at 16 weeks to confirm LITA to LAD graft patency. At 12 weeks, all animals had confirmed proximal LAD stenosis greater than $70 \%$ at the site of the occluder (Figure 2, $A$ ). The animals underwent revascularization with LITA to LAD, and at 16 weeks all 7 animals had patent LITA to LAD graft anastomoses confirmed by MDCT (Figure 2, $B$ ). Graft patency finding was proved accurate in all animals at necropsy by direct instrumentation with $1.5-\mathrm{mm}$ probe.

At the terminal procedure, blood flow had returned to normal at rest in the revascularized hibernating myocardium relative to the remote region after revascularization (Figure 3). Blood flow during dobutamine infusion, however, was reduced by $30 \%$ relative to normal. This region correlated with the area with persistently depressed myocardial function on TTE during dobutamine infusion (Figure 3).

Because myocardial function and blood flow did not return to normal in the LAD territory relative to the remote territory at high work load, we assessed the tissue for evidence of infarction or microvascular alterations. Triphenyl tetrazolium chloride stain confirmed the viability of the revascularized hibernating myocardium in all animals. To assess for fibrosis or abnormal vascularity, Masson trichrome
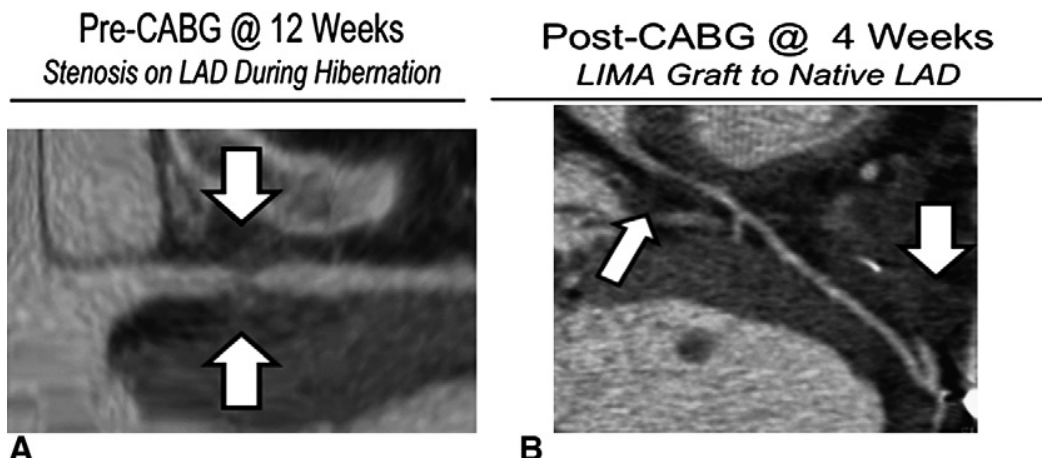

FIGURE 2. A. Multidetector computed tomographic image of left anterior descending coronary artery $(L A D)$ stenosis (white arrows) before revascularization surgery $(C A B G)$. B, Multidetector computed tomography illustrates proximal stenosis of left anterior descending coronary artery (LAD, thin arrow) with patent left internal thoracic artery (LIMA) to left anterior descending coronary artery anastomosis (thick arrow) at 4 weeks after revascularization (CABG). 
Blood Flow Analysis In Revascularized Animals

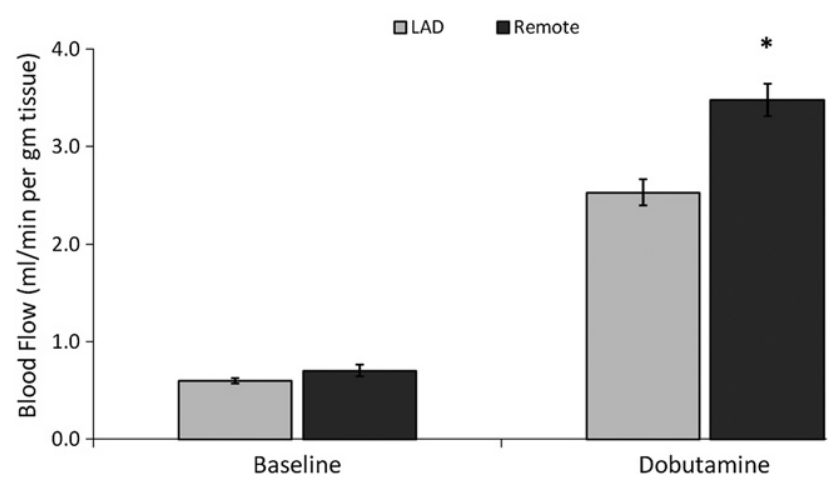

FIGURE 3. Fluorescently labeled color microspheres demonstrated return of blood flow in revascularized myocardium to baseline flow at rest but with significant reduction remaining during dobutamine infusion at $40 \mu \mathrm{g} /(\mathrm{kg}$. $\min )$ for 5 minutes $(\mathrm{n}=7)$ in left anterior descending coronary artery $(L A D)$ territory relative to remote territory. Asterisk indicates $P<.05$.

stain of the remote myocardium was compared with that in the revascularized hibernating LAD territory. We did not find significant fibrosis or alteration in microvascular architecture (Figure 4).

Because of the reduced function and blood flow with dobutamine infusion despite revascularization and the absence of scar, we analyzed mitochondrial oxygen consumption and proteomic expression as potential mechanisms of impaired oxygen use. We found that the RCI in the revascularized LAD region was lower than that in the remote region (Table 1). We compared this finding with the RCI from hibernating myocardium ${ }^{12}$ and found that the RCI was improved in the revascularized LAD myocardium relative to hibernating LAD myocardium.

By using iTRAQ analysis to identify and quantify the alterations in the ETC mitochondrial proteins, we found consistent reductions in expression of the mitochondrial oxidative phosphorylation protein subunits in complexes I, II, III, IV, and V (Table 2) in the revascularized hibernating myocardium relative to normal swine myocardium.

\section{DISCUSSION}

The principal findings of our study are that successful coronary artery revascularization of hibernating myocardium in a well-characterized swine model of chronic myocardial ischemia does not normalize maximal oxygen consumption despite successful grafting with LITA conduit. Basal contractile function and blood flow improved after revascularization, but not to a normal state with dobutamine stress. Basal contraction may relate to persistent changes within the mitochondrial inner membrane. Both key ETC proteins and RCI were reduced, suggesting that there is a persistent downregulation of the myocardial contractile capacity that limits oxygen consumption with expenditure. Similarly, we have shown that myocardial tissue adapts favorably to repetitive supply-demand challenges by reducing sources of oxidant stress, but ultimately at the expense of maximal oxygen consumption and expenditure. ${ }^{11}$

The importance of mitochondrial function to cardioprotection has been demonstrated by several observations. Isolated mitochondria from preconditioned myocardium retain the capacity to generate adenosine triphosphate after sustained ischemia and reperfusion ${ }^{16}$ and are resistant to in vitro anoxia and reoxygenation injury. ${ }^{17-19}$ In investigations of hibernating myocardium, Page and associates ${ }^{20}$ have demonstrated that myocardial hibernation is associated with reductions in key mitochondrial proteins associated with the ETC. With a proteomic method of total cellular proteins, the importance of the decreased protein expression was evident, with reduced enzymatic activity measurements of key mitochondrial proteins, such as pyruvate dehydrogenase and cytochrome c oxidase. The reductions in mitochondrial ETC proteins and contractile function 5 months after placement of a similar coronary artery constrictor suggest that the decreased expression of ETC proteins is related to the reduced blood flow. Whereas reductions in adenosine triphosphate synthase also correlate with the reduction in subendocardial blood flows in the hibernating myocardium, at the same time overexpression of several cytosolic proteins has been observed, including the antioxidant enzyme

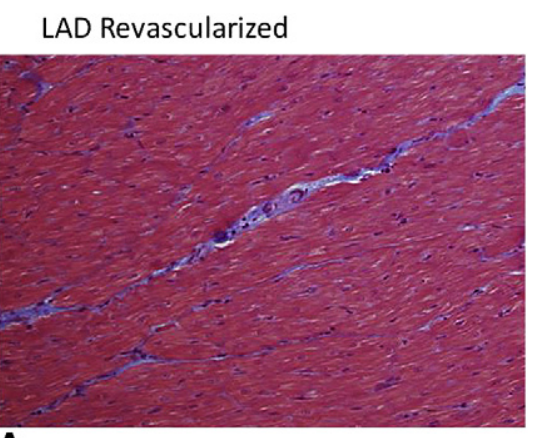

A
Remote Revascularized

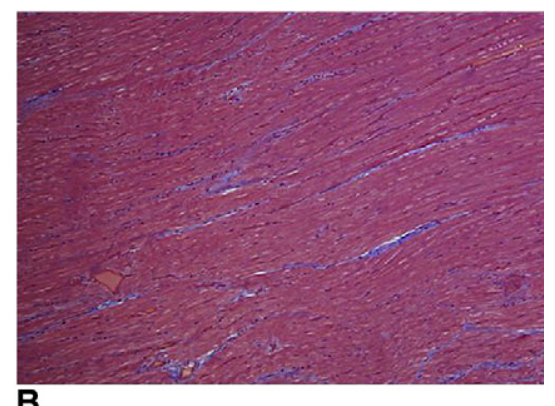

B

FIGURE 4. Revascularized left anterior descending coronary artery $(L A D)$ hibernating myocardial tissue (A) and revascularized remote myocardial tissue (B) stained with Masson trichrome stain (original magnification $\times 20$ ). 
TABLE 1. Respiratory Control Index of the revascularized left anterior descending coronary artery region and the control remote region in chronic hibernating (nonrevascularized) $\operatorname{animals}^{12}(n=10)$ and revascularized animals $(n=5)$

\begin{tabular}{lccr}
\hline & LAD & Remote & \multicolumn{1}{c}{$\boldsymbol{P}$ value* } \\
\hline Chronic hibernating swine & $2.75 \pm 0.15$ & $3.70 \pm 0.05$ & $<0.005$ \\
Revascularized swine & $3.87 \pm 0.33$ & $4.66 \pm 0.30$ & 0.035 \\
\hline
\end{tabular}

LAD, Left anterior descending coronary artery. ${ }^{*}$ By $t$ test.

superoxide dismutase 1 . Interestingly, the temporal increase in the antioxidant proteins coincides with the time that apoptosis is diminished in their model. ${ }^{21} \mathrm{~A}$ logical hypothesis is that superoxide production stimulates a stress response, which reduces subsequent reactive oxygen species production and thus promotes a survival pathway within the mitochondria. ${ }^{22}$ Such mitochondrial adaptations preserve viability in hibernating myocardium at the expense of energy production, and it is unknown to what extent these changes are reversible with revascularization.

Accordingly, we sought a suitable model of hibernation in swine to test whether successful revascularization could reverse the mitochondrial alteration to key ETC proteins. Somewhat surprisingly, despite restoration of baseline blood flow and viable myocardium, mitochondrial ETC protein expression remained depressed. The degree of alteration may negatively affect myocardial functional recovery, especially during increased workload. We found with a dobutamine infusion that although the blood flow did increase in the revascularized region, the maximal blood flow increase was $30 \%$ less than that in the remote region. Although we only evaluated recovery at 4 weeks after revascularization, and clinical experience suggests that recovery from hibernation may continue for several months after revascularization, these results are intriguing and support clinical observations that complete recovery of hibernating myocardium is not guaranteed, despite successful revascularization.

The novelty of this study is that it is the first report, to our knowledge, of bypass grafting to a coronary artery in a suitable animal model of myocardial hibernation. Our large animal model mimics the clinical scenario of surgical revascularization of hibernating myocardium and therefore is relevant to patient care. Several longitudinal studies of patients with ischemic cardiomyopathy have demonstrated that progressive heart failure may persist despite successful revascularization. ${ }^{23-25}$ These clinical studies have not provided a unifying concept to predict when the decrease in contractile function in chronic, persistent myocardial ischemia may ultimately recover.

We have demonstrated for the first time in a large animal model that an aggressive intervention that restores blood flow to the hibernating myocardium is feasible and allows simultaneous mitochondrial proteomic analysis and myocardial assessment. We found in a swine model of
TABLE 2. Isobaric tags for relative and absolute quantification results of hibernating mitochondria following revascularization compared to normal myocardium $(n=7)$

\begin{tabular}{|c|c|c|}
\hline Protein type and name & $\begin{array}{c}\text { Revascularized } \\
\text { vs control } \\
\end{array}$ & $P$ value \\
\hline \multicolumn{3}{|l|}{ Oxidative phosphorylation } \\
\hline \multicolumn{3}{|l|}{ Complex I } \\
\hline NADH dehydrogenase & $0.58 \pm 0.056$ & .0003 \\
\hline NADH dehydrogenase (ubiquinone) $1 \alpha$ & $0.66 \pm 0.040$ & .0001 \\
\hline NADH dehydrogenase (ubiquinone) $1 \beta$ & $0.66 \pm 0.073$ & .0036 \\
\hline \multicolumn{3}{|l|}{ Complex II } \\
\hline Succinate dehydrogenase subunit A & $0.71 \pm 0.080$ & .0117 \\
\hline \multicolumn{3}{|l|}{ Complex III } \\
\hline Ubiquinol-cytochrome c reductase & $0.78 \pm 0.080$ & .0356 \\
\hline $\begin{array}{l}\text { Ubiquinol-cytochrome } \mathrm{c} \text { reductase, } \\
\text { Rieske polypeptide } 1\end{array}$ & $0.65 \pm 0.035$ & $<.0001$ \\
\hline \multicolumn{3}{|l|}{ Complex IV } \\
\hline Cytochrome c oxidase subunit $6 \mathrm{~B}$ & $0.57 \pm 0.063$ & .0005 \\
\hline Cytochrome $\mathrm{c}$ oxidase subunit $\mathrm{Vb}$ & $0.68 \pm 0.068$ & .0031 \\
\hline Cytochrome $\mathrm{c}$ oxidase subunit VIc & $0.69 \pm 0.050$ & .0008 \\
\hline \multicolumn{3}{|l|}{ Complex V } \\
\hline ATP synthase $\mathrm{F}_{0}$ & $0.59 \pm 0.044$ & $<.0001$ \\
\hline $\begin{array}{l}\text { ATP synthase, } \mathrm{H}^{+} \text {transporting, } \\
\text { mitochondrial } \mathrm{F} 1 \text { complex, } \alpha\end{array}$ & $0.66 \pm 0.079$ & .0047 \\
\hline $\begin{array}{l}\text { ATP synthase, } \mathrm{H}^{+} \text {transporting, } \\
\text { mitochondrial } \mathrm{F} 1 \text { complex, } \beta\end{array}$ & $0.66 \pm 0.053$ & .0007 \\
\hline $\begin{array}{l}\text { ATP synthase, } \mathrm{H}^{+} \text {transporting, } \\
\text { mitochondrial F1 complex, } \delta\end{array}$ & $0.53 \pm 0.071$ & .0006 \\
\hline $\begin{array}{l}\text { ATP synthase, } \mathrm{H}^{+} \text {transporting, } \\
\text { mitochondrial F1 complex, } \gamma\end{array}$ & $0.74 \pm 0.055$ & .0028 \\
\hline
\end{tabular}

$\overline{N A D H}$, Nicotinamide adenine dinucleotide (reduced form); $A T P$, adenosine triphosphate.

myocardial hibernation in which regional blood flow and contraction were reduced in the absence of necrosis that successful bypass with LITA graft to the involved LAD artery improves but does not normalize regional function. The inability to recruit maximal blood flow at high work states and the reduced expression of key ETC proteins within involved mitochondria together suggest that persistent mitochondrial adaptations within hibernating myocardium may underlie the mechanisms of persistent myocardial dysfunction in successfully revascularized hibernating heart tissue. Future studies should address whether restoration of mitochondrial protein expression, either pharmacologically or with cell-based therapies, might modify the persistent contractile abnormalities within chronically ischemic heart tissue.

\section{References}

1. Rahimtoola SH. The hibernating myocardium. Am Heart J. 1989;117:211-21.

2. Fallavollita JA, Malm BJ, Canty JM Jr. Hibernating myocardium retains metabolic and contractile reserve despite regional reductions in flow, function, and oxygen consumption at rest. Circ Res. 2003;92:48-55.

3. Mills I, Fallon JT, Wrenn D, Sasken H, Gray W, Bier J, et al. Adaptive responses of coronary circulation and myocardium to chronic reduction in perfusion pressure and flow. Am J Physiol. 1994;266(2 Pt 2):H447-57.

4. McFalls EO, Baldwin D, Palmer B, Marx D, Jaimes D, Ward HB. Regional glucose uptake with hypoperfused swine myocardium as measured by positron emission tomography. Am J Physiol. 1997;272(1 Pt 2):H343-9. 
5. Fallavollita JA, Perry BJ, Canty JM Jr. 18F-2-deoxyglucose deposition and regional flow in pigs with chronically dysfunctional myocardium. Evidence for transmural variations in chronic hibernating myocardium. Circulation. 1997; 95:1900-9.

6. Fallavollita JA, Jacob S, Young RF, Canty JM Jr. Regional alterations in $\mathrm{SR} \mathrm{Ca}^{2+}$ ATPase, phospholamban, and HSP-70 expression in chronic hibernating myocardium. Am J Physiol. 1999;277(4 Pt 2):H1418-28.

7. Bito V, van der Velden P, Claus P, Dommke C, van Lommel A, Mortelmans L, et al. Reduced force generating capacity in myocytes from chronically ischemic hibernating myocardium. Circ Res. 2007;100:229-37.

8. Lim H, Fallavollita JA, Hard R, Kerr CW, Canty JM Jr. Profound apoptosismediated regional myocyte loss and compensatory hypertrophy in pigs with hibernating myocardium. Circulation. 1999;100:2380-6.

9. Murry CE, Jennings RB, Reimer KA. Preconditioning with ischemia: a delay of lethal cell injury in ischemic myocardium. Circulation. 1986;74:1124-36.

10. Dzeja PP, Holmuhamedov EL, Ozcan C, Pucar D, Jahangir A, Terzic A. Mitochondria: gateway for cytoprotection. Circ Res. 2001;89:744-6.

11. Kelly RF, Sluiter W, McFalls EO. Hibernating myocardium: is the program to survive a pathway to failure? Circ Res. 2008;102:3-5.

12. McFalls EO, Sluiter W, Schoonderwoerd K, Manintveld OC, Lamers JM, Bezstarosti K, et al. Mitochondrial adaptations within chronically ischemic swine myocardium. J Mol Cell Cardiol. 2006;41:980-8.

13. McFalls EO, Liem D, Schoonderwoerd K, Lamers J, Sluiter W, Duncker D. Mitochondrial function: the heart of myocardial preservation. J Lab Clin Med. 2003;142:141-8.

14. Kassie F, Anderson LB, Higgins L, Pan Y, Matise I, Negia M, et al. Chemopreventive agents modulate the protein expression profile of 4-(methylnitrosamino)1-(3-pyridyl)-1-butanone plus benzo[a]pyrene-induced lung tumors in $\mathrm{A} / \mathrm{J}$ mice. Carcinogenesis. 2008;29:610-9.

15. Lund TC, Anderson LB, McCullar V, Higgins L, Yun GH, Grzywacz B, et al. iTRAQ is a useful method to screen for membrane-bound proteins differentially expressed in human natural killer cell types. J Proteome Res. 2007;6:644-53.

16. Fryer RM, Eells JT, Hsu AK, Henry MM, Gross GJ. Ischemic preconditioning in rats: role of mitochondrial $\mathrm{K}(\mathrm{ATP})$ channel in preservation of mitochondrial function. Am J Physiol Heart Circ Physiol. 2000;278:H305-12.

17. Ziemba EA, McFalls EO, Cabrera JA, Sikora J, Crampton M, Ward HB, Kelly RF. A favorable energetic state is observed during low flow myocardial ischemia in the Second Window of Preconditioning (SWOP) in swine despite an increased expression of uncoupling protein-2. Society of University Surgeons. 2010; Abstract No. 515

18. Ozcan C, Bienengraeber M, Dzeja PP, Terzic A. Potassium channel openers protect cardiac mitochondria by attenuating oxidant stress at reoxygenation. Am J Physiol Heart Circ Physiol. 2002;282:H531-9.

19. Ozcan C, Holmuhamedov EL, Jahangir A, Terzic A. Diazoxide protects mitochondria from anoxic injury: implications for myopreservation. J Thorac Cardiovasc Surg. 2001;121:298-306.

20. Page B, Young R, Iyer V, Suzuki G, Lis M, Korotchkina L, et al. Persistent regional downregulation in mitochondrial enzymes and upregulation of stress proteins in swine with chronic hibernating myocardium. Circ Res. 2008;102:103-12.

21. Depre C, Kim SJ, John AS, Huang Y, Rimoldi OE, Pepper JR, et al. Program of cell survival underlying human and experimental hibernating myocardium. Circ Res. 2004;95:433-40.

22. Minners J, Lacerda L, McCarthy J, Meiring JJ, Yellon D, Sack M. Ischemic and pharmacological preconditioning in Girardi cells and C2C12 myotubes induce mitochondrial uncoupling. Circ Res. 2001;89:787-92.

23. Heusch G, Schultz R, Rahimtoola SH. Myocardial hibernation: a delicate balance. Am J Physiol Heart Circ Physiol. 2005;288:H984-99.

24. Depré C, Vanoverschelde JL, Melin JA, Borgers M, Bol A, Ausma J, et al. Structural and metabolic correlates of the reversibility of chronic left ventricular ischemic dysfunction in humans. Am J Physiol. 1995;268(3 Pt 2):H1265-75.

25. Vanoverschelde JL, Wijns W, Depré C, Essamri B, Heyndrickx GR, Borgers M, et al. Mechanisms of chronic regional postischemic dysfunction in humans. New insights from the study of noninfarcted collateral-dependent myocardium. Circulation. 1993;87:1513-23.

\section{Discussion}

Dr Keith Horvath (Bethesda, Md). Thank you, Dr Kelly, excellent presentation of some very intriguing work. I have just a few questions.
The results that you show document that there is some persistent dysfunction particularly at the mitochondrial level after revascularization. Are you convinced that this is because of that at the cellular level or is it possible that there still is a blood flow problem? You've documented that the baseline resting blood flow returns to normal but with dobutamine stress there is a difference. Again, is it a result of the flow in or is it as a result of the mitochondrial dysfunction?

From a mechanistic point of view, what do you postulate is causing this? One of the main reasons may be apoptosis, and do you have any data that supports that as the principal mechanism?

And then, finally, from a clinical implication point of view, is there something that we should be doing in the operating room or postoperatively to prevent this from happening? And then to delve somewhat into a controversial area, is this because these were performed off-pump instead of on-pump?

Dr Kelly. Thank you for your questions.

To begin with, we did consider other reasons for decreased perfusion. We looked at the vascular reactivity in the resistant vessels of the myocardium and what we found is that the contractility remained normal in the LAD versus circumflex vessels. What we have not yet investigated, and our future studies are designed to study, is the ability of the LAD vessels to relax normally. I think when we perform a dobutamine stress test, what may be happening is a signaling for dilation to which the vasculature in the hibernating tissue is unable to respond. That will be for future considerations.

When we looked at possible mechanisms as to why the myocardial function remains abnormal, we did not look specifically for apoptosis within the tissue. But looking at the histology and the electron microscopy, we have found that these cells and mitochondrial remain normal in appearance and have not noticed a significant presence of necrosis or fibrosis.

As the cellular and tissue structure remained intact following chronic ischemia and revascularization, we investigated other possible mechanisms by which the cells fail to recover upon revascularization and focused on oxygen utilization in the mitochondria. With uncoupling of oxidative phosphorylation, the mitochondria are unable to maximally respond to the oxygen delivered and ATP production remains impaired. When we looked at the mitochondrial function using Clark's electrode, the respiratory control index in hibernating tissue remained depressed. When we looked at our revascularized hibernating tissue compared to remote, it remained depressed as well. This finding suggests that the mitochondria themselves remain dysfunctional compared to normal tissue.

And finally, I agree with you, the off-pump technique could clearly impact the microthrombus occurring in these animal models. In particular for this model, pigs can be very thrombogenic. However, for technical support and recovery reasons, it was better to do the procedure off-pump and we did not see any evidence of thrombus intraoperatively or evidence of infarction at the time of the terminal study. Also, we aggressively heparinized the animals to prevent thrombosis

Dr Paul Kurlansky (Miami, Fla). Beautiful piece of work, it was very intriguing. I had 3 questions.

One was, clearly you seem to be postulating that there is some sort of trigger which is set off in the hibernating myocardium which is not readily reversed. Do you have any idea specifically what this trigger is mechanistically and how we therefore might address it? 
Secondly, another approach might be whether or not you had looked at the effect of a preconditioning, preconditioner, even postconditioning, and if it somehow affects the ability of revascularization to turn this trigger the other direction.

And the third question is, it's very difficult to do the experiments you're doing and it's even more difficult to do them for prolonged survival; however, over time, do you have any idea if after several months does the trigger get reversed with adequate revascularization?

Dr Kelly. We don't know what the trigger for hibernation is but agree that there is some alteration that occurs in the mitochondria that moves it into survival mode rather than necrosis or apoptosis. It is possibly an upregulation of uncoupling protein but the trigger for this response is unclear. Discovering that trigger, and the mechanism for reversal, is an important part of our investigations going forward.
We have looked at the spectrum of preconditioning in relation to hibernation, but not yet in the revascularization model. However, if you look at what we've created, we seem to have transitioned from hibernation to stunned myocardium, where you have normal blood flow yet depressed function. If we continue on that spectrum, we may indeed see that finally at a year the myocardial function would return to normal.

We plan to allow for more prolonged recovery in this animal model to study the recovery of the myocardium over time. This will mimic the clinical experience of continued recovery over several months. For me, as a clinician, the question would be: How can I help that recovery occur more quickly following revascularization? We are looking at manipulating possible triggers using pharmacologic adjuvant therapy as well as using stem cells to permit more rapid recovery. Those are our speculations for the future directions using this model. 